\title{
An Application of Autoregressive Extreme Value Theory to Cryptocurrencies
}

\begin{abstract}
We study the tails' behavior of four major Cryptocurrencies (Bitcoin, Litecoin, Ethereum, and Ripple) by employing the Autoregressive Fréchet model for conditional maxima. Using five-minutehigh-frequency data, we report time-evolving tails as well as provide a straightforward measure of tails asymmetry for positive and negative intra-day returns. We find that only Bitcoin has a notable more massive tail for positive returns asymmetry while the remaining three Cryptocurrencies have a general tendency towards more massive negative intra-day tails. All considered Cryptocurrencies depict lighter tails as the market matures.
\end{abstract}

Keywords: Cryptocurrencies, Bitcoin, Extreme value theory, Time-varying tails, the Expected shortfall 


\section{Introduction}

Cryptocurrencies attract the increasing attention of policymakers, practitioners, and academics. ${ }^{1}$ Recent studies examined whether Bitcoin possesses a safe haven property (e.g., Smales, 2018), hedging properties (Aysan et al., 2019; Demir et al., 2018; Gozgor et al., 2019) how elaborate portfolio management techniques fit Cryptocurrencies' setting (e.g., Platanakis \& Urquhart, 2019; Poyser, 2019) and if Bitcoin and Gold fundamentally share similar properties (e.g., Klein et al., 2018). Literature also investigated the appropriateness of various risk measures and quantitative models for Cryptocurrencies (e.g., Peng et al., 2018; Trucios, 2019), among other topics. In particular, and closely related to our paper, Gkillas \& Katsiampa (2018) highlight the value of the tail index measure of Cryptocurrencies for risk assessment by various financial audiences. They provide fundamental insights into Extreme Value Theory (henceforth EvT) tail shapes for five Cryptocurrencies, including Bitcoin (BTC), Litecoin (LTC), Ethereum (ETH), Ripple (XRP) and Bitcoin Cash.

In this paper, we contribute to the academic literature by building upon Gkillas \& Katsiampa's(2018) work and complementing previous EvT findings with Autoregressive Conditional Fréchet (ACF) model for intra-day maxima of Zhao et al. (2018). ACF model of Zhao et al. (2018) has several notable differences from the Generalised Pareto Distribution (GPD) with the peaks-over-threshold approach for modeling extremes as in Gkillas \& Katsiampa (2018). EvT GPD and its common GARCH filtered alternative of McNeil \& Frey $(2000)^{2}$ both provide a static view on the tail shapes of positive and negative Cryptocurrency returns. On the other hand, with our analysis, we can investigate the time-varying scale (volatility) alongside time-varying tail indices with a fully autoregressive EvT model. Gkillas \& Katsiampa (2018) characterize Cryptocurrencies as speculative

\footnotetext{
${ }^{1}$ See Corbet et al. (2019) for a review of the empirical literature based on the essential topics that have been associated with the market for Cryptocurrencies since their development as a financial asset.

${ }^{2}$ In the context of Cryptocurrencies, this EVT approach is also employed by Feng et al. (2018).
} 
assets and, therefore, it is imperative to investigate the time evolution of their tails and attempt to unpacking their speculative nature in more detail. For example, similar to Chaim \& Laurini (2018), we document diminishing Cryptocurrencies' volatility. However, at the same time, we observe a notable tendency of higher tail indices (lighter tails) in positive and negative returns of BTC, LTC, ETH, and XRP with time. It is also essential to understand whether and how time conditional tail indices of Cryptocurrencies are similar to tails of conventional currencies and other financial assets utilizing the range of techniques employed in Klein et al. (2018). Besides, we contribute to the literature by performing a simple time-varying tail asymmetry analysis. We find that BTC intra-day extremes consistently suggest different speculative behavior from LTC, ETH, and XRP. This finding is valuable for trading (e.g., Cheng et al., 2019) or portfolio (e.g., Platanakis \& Urquhart, 2019) relevant investigations.

We also contribute to the literature by providing the first empirical applications of the ACF model and block maxima EVT division to the widely accepted five-minute frequency data standard (e.g., Liu et al., 2015) for Cryptocurrencies. Zhang et al. (2019) also consider intra-day data; however, they only apply the GPD EVT model similar to Gkillas \& Katsiampa (2018) and concentrate on the one-hour data frequency. Cryptocurrencies represent a fruitful field for an empirical test of the ACF model and, therefore, we complement our tail shapes oriented investigation with a basic in-sample expected shortfall test. We find the mediocre performance of the model in our setting, yielding consistently higher rates of the actual conditional five-minute maxima exceedances than expected.

The rest of the paper is structured as follows: Section 2 outlines the ACF model of Zhao et al. (2018), then this section describes our data. Section 3 presents the estimation results, and Section 4 summarizes conducted work in the concluding remarks section. 


\section{Methodology and Data}

\subsection{Autoregressive Conditional Fréchet Model}

For the Data Generating Process (DGP) outlined by the below Probability Density Function (PDF)

$$
f\left(Q_{t} \mid \mu, \sigma_{t}, \alpha_{t}\right)=\frac{\alpha_{t} \sigma_{t}^{\alpha_{t}}}{\left(Q_{t}-\mu\right)^{\alpha_{t}+1}} \exp \left[-\sigma_{t}^{\alpha_{t}}\left(Q_{t}-\mu\right)^{-\alpha_{t}}\right],
$$

with

$$
\begin{aligned}
Q_{t} & =\mu+\sigma_{t} Y_{t}^{1 / \sigma_{t}} ; \\
\log \sigma_{t} & =\beta_{0}+\beta_{1} \log \sigma_{t-1}-\beta_{2} \cdot \exp \left(-\beta_{3} \cdot Q_{t-1}\right) ; \\
\log \alpha_{t} & =\theta_{0}+\theta_{1} \log \alpha_{t-1}+\theta_{2} \cdot \exp \left(-\theta_{3} \cdot Q_{t-1}\right),
\end{aligned}
$$

where $Y_{t}$ denotes a sequence of independent and identically distributed Fréchet random variables, Zhao et al. (2018) impose $0<\beta_{1} \neq \theta_{1}<1, \beta_{2}>0, \beta_{3}>0, \theta_{2}>0$ and $\theta_{3}>0$ constraints and establish an EvT conditional daily maxima model for intra-day price data. Moreover, with recursions in Eq.(2), it is intrinsic to suggest a parsimonious measure of time-varying tail asymmetry outlined by:

$$
T A S_{t}=\frac{2 \alpha_{t}^{-}-2 \alpha_{t}^{+}}{\alpha_{t}^{-}+\alpha_{t}^{+}}
$$

Eq. (3) is defined for more informative tail dynamics investigation. Note that measure in Eq. (3) is just a simple adaption of the asymmetry developed for volatility spillovers investigation with high-frequency data as in Apergis et al. (2017). Since the lower is the value of the tail index, the heavier are the tails and, hence, the higher is the likelihood to observe positive or negative intra-day maxima, we reformulate measure in Apergis et al. (2017), so that 
asymmetry measure in Eq.(3) reflects reverse nature of EvT tail indices. To clarify, if negative (positive) intra-day conditional maxima tail is heavier than the positive (negative) tail, then Eq. (3) yields a negative (positive) value, respectively. Finally, we complete our EvT investigation by in-sample tests for Expected Shortfall (ES) at confidence levels as in McNeil \& Frey (2000). For McNeil \& Frey's (2000) test for ES with DGP in Eq. (1), Value-at-Risk (VaR) is respectively given by:

$$
\operatorname{VaR}_{t}(\lambda)=\mu+\sigma_{t}(-\log \lambda)^{-1 / \alpha},
$$

while ES is outlined by:

$$
\operatorname{ES}^{t}(\lambda)=\frac{\mu+\sigma_{t}}{1-\lambda} \int_{\lambda}^{1}[-\log z]^{-1 / \alpha} d z,
$$

Where $\lambda \in(0,1)$.

\subsection{Data and Preliminary Findings}

We convert Bitfinex one-minute BTC, LTC, ETH, and XRP - USD exchange rates to five-minute frequency data and obtain daily maxima for positive and negative (minima) returns. Exchange series we employ are available on Kaggle ${ }^{3}$ and include observations from the 1st of April 2013 for BTC, 19th of May 2013 for LTC, 9th of March 2016 for ETH and 19th of May 2017 for XRP until the 2nd of August 2019. Although, in the literature, Bitfinex exchange data is commonly employed due to the data available through its public API (see, e.g., Borri, 2019), it is essential to highlight that the exchange stopped its operations several times due to the events described in Corbet et al. (2019). An alternative, and more consistent Cryptocurrency exchange, with similar data access level through the public API and higher trading volumes is Binance. However, this exchange is relatively recent and could limit our analysis of evolving tail behavior. Corbet et al. (2019) point out that rates at different exchanges may differ for the same Cryptocurrency, but are

\footnotetext{
${ }^{3}$ Courtesy of Carsten Klein.
} 
highly interrelated. Therefore, we also run the Bitstampt exchange BTC-USD pair available on Kaggle as an empirical check. This exchange is also frequent in the relevant literature (e.g., Trucios, 2019), and our sample includes BTC observations from the 31st of December 2011 to the 2nd of August 2019. We provide obtained minima and maxima for BTC and LTC in Figure 1 and ETH and XRP in Figure 2. Bitstamp BTC data is illustrated in Figure 3.

[Insert Figure 1, 2 and 3 here]

From Figures 1, 2, and 3, a general diminishing trend in minima and maxima values can be noted. We also identify phases of rapid growth and decline of Cryptocurrencies as well as their return to the current price levels (being less apparent). Identifying a diminishing trend in the daily maxima or minima does not necessarily imply time-varying EVT tails since the timevariation can capture a notable portion of time-variation in the intra-day data in scale or volatility parameter. Indeed, diminishing scale parameters can be observed for both positive and negative blocks (Figures 1 and 2). Overall, according to Figures 1 and 2, the scale parameters of BTC and LTC have significantly diminished, but the scale parameters of ETH and XRP only have a slight decrease.

For ETH, the scale for negative and positive returns does not exhibit a noticeable decay over time (Figure 2), but we observe a relevant and general pattern of price evolution for Cryptocurrencies. On the other hand, fluctuations in ETH time-varying tail indices are evident, highlight ups and downs in the price changes, and demonstrate a tendency for both lighter left and right tails. Similar observations can be made by analyzing tail indices of other Cryptocurrencies in our pool. BTC thinner tails tendency is less notable on the Bitfinex exchange; however, a more extended sample perspective from Bitstamp unpacks this evidence for BTC. Overall, we empirically demonstrate an analytical conclusion by Gkillas \& Katsiampa (2018) 
that as the market for Cryptocurrencies matures, we may expect these assets are exhibiting different tail behavior.

\section{Results and Discussion}

We aggregate the evolution of tail indices into box-plots and assess left and right tails asymmetries. For XRP, the riskiest Cryptocurrency in the pool, we note that, on average, the lower tail is more substantial than its upper tail. This issue is a common observation of financial assets' returns. On the other hand, relative symmetry is observed for LTC and ETH, while BTC stands out with more massive tails for positive than its negative returns. Although BTC asymmetry observation is uniform in Figures 1 and 3, the range of the tail indices is different for Bitstamp and Bitfinex data. Since BTC median tail indices are similar, and Bitstamp sample is more substantial, and this can be explained by the highlights of Corbet et al. (2019) on Cryptocurrencies' differences across different exchanges. We draw various conclusions on speculative behavior among Cryptocurrencies by investigating tail asymmetry over time. Consistent positive asymmetry for BTC suggests that long intra-day speculative positions in BTC are typically less risky, whereas short speculative positions have less extreme exposure for LTC and XRP. ETH has a mixed asymmetry outlook. It has correct and neutral tail asymmetries over the Cryptocurrencies price expansions cycles and negative asymmetry over flat trading and price downturns periods.

Furthermore, we report the in-sample test results for ES in Table 1. We obtain an alarmingly higher number of actual exceedances than expected, with the only exception being the 95\% risk confidence level for LTC. ACF model approximates accepted mean levels of obtained 
VaR violations for the majority of the risk levels and Cryptocurrencies, with the notable exception of LTC. Such LTC results are the least expected since it is not the most heavy-tailed among the considered Cryptocurrencies. Our other exchange check provides very similar violations and better ES outlook. However, given the results we obtain for the number of block minima and maxima violations, we cannot conclude that obtained test results are satisfactory.

[Insert Table 1 around here]

\section{Concluding Remarks}

Cryptocurrencies are among the fast-evolving speculative assets. In this paper, we provide empirical evidence of time-varying tails and asymmetry in the indices characterizing the extremes of these assets. Our work is a logical extension of the analysis conducted by Gkillas \& Katsiampa (2018) and Feng et al. (2018) from the perspective of data frequency and the models employed. Although we do not find strong evidence that the ACF model provides convincing results for intra-day exposure modeling, this does not necessarily imply that EVT is not fit for risk approximation of Cryptocurrencies. Gkillas \& Katsiampa (2018) and Feng et al. (2018) investigations do not cover VaR or ES backtesting. Hence, we believe this is a valuable direction for further research since it is problematic performing empirical comparisons to the EvT models employed in the previous studies at this stage. On the other hand, Feng et al. (2018) suggest that Cryptocurrencies EvT tails decay may have changed from Fréchet to Weibull type. Therefore, our Fréchet restricted assumption on the DGP may not be sufficient, and it is worthwhile considering more flexible Generalized Extreme Value (GEV) distribution (e.g., Chavez-Demoulin et al., 2016) for better backtesting outcomes. For now, high-frequency exposure of Cryptocurrencies may be modeled with approaches empirically tested by Trucios (2019), but we encourage researchers to challenge or update our findings as more data on Cryptocurrencies become available from more stable and more prominent exchanges such as Binance. 


\section{References}

Apergis, N., Barunik, J., \& Lau, M.C.K. (2017). Good volatility, bad volatility: What drives the asymmetric connectedness of Australian electricity markets? Energy Economics, 66, 108115.

Aysan, A.F., Demir, E., Gozgor, G. \& Lau, C.K.M. (2019). Effects of Geopolitical Risks on the Bitcoin Returns and Volatility. Research in International Business and Finance, 47, 511-518.

Borri, N. (2019). Conditional tail-risk in cryptocurrency markets. Journal of Empirical Finance, 50, $1-19$.

Chaim, P., \& Laurini, M. P. (2018). Volatility and return jumps in Bitcoin. Economics Letters, 173, $158-163$.

Chavez-Demoulin, V., Embrechts, P., \& Hofert, M. (2016). An extreme value approach for modeling operational risk losses depending on covariates. Journal of Risk and Insurance, 83 (3), 735-776.

Cheng, Q., Liu, X., \& Zhu, X. (2019). Cryptocurrency momentum effect: DFA and MF-DFA analysis. Physica A: Statistical Mechanics and its Applications, 526, 120847.

Corbet, S., Lucey, B., Urquhart, A., \& Yarovaya, L. (2019). Cryptocurrencies as a financial asset: A systematic analysis. International Review of Financial Analysis, 62, 182-199.

Demir, E., Gozgor, G., Lau, C.K.M. \& Vigne, S. (2018). Does Economic Policy Uncertainty Predict the Bitcoin Returns? An Empirical Investigation. Finance Research Letters, 26, 145-149.

Feng, W., Wang, Y., \& Zhang, Z. (2018). Can cryptocurrencies be a safe haven: A tail risk perspective analysis. Applied Economics, 50 (44), 4745-4762.

Gkillas, K., \& Katsiampa, P. (2018). An application of extreme value theory to Cryptocurrencies. Economics Letters, 164, 109-111. 
Gozgor, G., Tiwari, A.K., Demir, E. \& Akron, S. (2019). The Relationship between Bitcoin Returns and Trade Policy Uncertainty. Finance Research Letters, 29, 75-82.

Klein, T., Thu, H.P., \& Walther, T. (2018). Bitcoin is not the New Gold-a comparison of volatility, correlation, and portfolio performance. International Review of Financial Analysis, 59, 105116.

Liu, L.Y., Patton, A. J., \& Sheppard, K. (2015). Does anything beat 5-minute RV? A comparison of realized measures across multiple asset classes. Journal of Econometrics, 187 (1), 293-311.

McNeil, A. J., \& Frey, R. (2000). Estimation of tail-related risk measures for heteroscedastic financial time series: An extreme value approach. Journal of Empirical Finance, 7 (3-4), $271-300$.

Peng, Y., Albuquerque, P. H. M., de Sa, J. M. C., Padula, A. J. A., \& Montenegro, M. R. (2018). The best of two worlds: Forecasting high-frequency volatility for cryptocurrencies and traditional currencies with Support Vector Regression. Expert Systems with Applications, 97, $177-192$.

Platanakis, E., \& Urquhart, A. (2019). Portfolio management with cryptocurrencies: The role of estimation risk. Economics Letters, 177, 76-80.

Poyser, O. (2019). Exploring the dynamics of Bitcoin's price: A Bayesian structural time series approach. Eurasian Economic Review, 9 (1), 29-60.

Smales, L. A. (2018). Bitcoin as a safe haven: Is it even worth considering? Finance Research Letters, 30, 385-393.

Trucios, C. (2019). Forecasting Bitcoin risk measures: a robust approach. International Journal of Forecasting, 35 (3), 836-847.

Zhang, Y., Chan, S., \& Nadarajah, S. (2019). Extreme value analysis of high-frequency cryptocurrencies. High Frequency, 2 (1), 61-69. 
Zhao, Z., Zhang, Z., \& Chen, R. (2018). Modeling maxima with autoregressive conditional Fréchet model. Journal of Econometrics, 207 (2), 325-351.

Table 1 Results of In-sample Backtesting for Expected Shortfall

\begin{tabular}{|c|c|c|c|c|c|c|c|c|c|c|}
\hline \multirow[t]{2}{*}{ Cryptocurrency } & \multicolumn{2}{|l|}{ BTC } & \multicolumn{2}{|l|}{ LTC } & \multicolumn{2}{|l|}{ ETH } & \multicolumn{2}{|l|}{ XRP } & \multicolumn{2}{|l|}{ BTC-B } \\
\hline & Negative & Positive & Negative & Positive & Negative & Positive & Negative & Positive & Negative & Positive \\
\hline $\mathrm{EE}$ & 90 & 90 & 88 & 88 & 37 & 37 & 15 & 15 & 114 & 114 \\
\hline \multirow{3}{*}{$\begin{array}{c}\mathrm{AE} \\
\mathrm{ES}_{p}(0.95) \\
\mathrm{EE}\end{array}$} & 104 & 90 & \multirow{3}{*}{$\begin{array}{c}69 \\
0.0016 \\
17\end{array}$} & \multirow{2}{*}{$\begin{array}{r}70 \\
0.0055\end{array}$} & \multirow{2}{*}{$\begin{array}{r}56 \\
0.0049\end{array}$} & 62 & \multirow{2}{*}{$\begin{array}{l}21 \\
0.0107\end{array}$} & 21 & 144 & 143 \\
\hline & 0.0515 & 0.1419 & & & & 0.1394 & & 0.7688 & 0.0759 & 0.4148 \\
\hline & 18 & 18 & & 17 & 7 & 7 & 3 & 3 & 22 & 22 \\
\hline \multirow{3}{*}{$\begin{array}{c}\mathrm{AE} \\
\mathrm{ES}_{p}(0.99) \\
\mathrm{EE}\end{array}$} & 36 & 21 & 30 & \multirow{2}{*}{$\begin{array}{r}28 \\
0.0229\end{array}$} & 25 & 19 & 12 & 4 & 47 & 39 \\
\hline & 0.0736 & 0.0067 & 0.0042 & & 0.1025 & 0.6279 & 0.5734 & 0.7766 & 0.0517 & 0.1008 \\
\hline & 9 & 9 & 8 & 8 & 3 & 3 & 1 & 1 & 11 & 11 \\
\hline \multirow{2}{*}{$\begin{array}{c}\mathrm{AE} \\
\mathrm{ES}_{p}(0.995)\end{array}$} & 24 & \multirow{2}{*}{$\begin{array}{r}18 \\
0.0133\end{array}$} & \multirow{2}{*}{$\begin{array}{r}21 \\
0.0049\end{array}$} & \multirow{2}{*}{$\begin{array}{r}17 \\
0.0129\end{array}$} & 18 & 9 & 8 & 2 & 30 & 23 \\
\hline & 0.1461 & & & & 0.1167 & 0.4089 & 0.8727 & 0.9984 & 0.0510 & 0.2667 \\
\hline
\end{tabular}

Notes: $\mathrm{EE}$ and $\mathrm{AE}$ denote Expected and Actual Exceedances, bootstrapped p-values exceeding 5\% threshold are highlighted in grey, while BTC-B denotes in-sample results for Bitcoin Bitstamp run. 
Figure 1

BTC and LTC Negative (Minima) and Positive Daily Maxima, EVT Time-varying Sca le Parameter, Tail Indices and Tail Asymmetry Measure
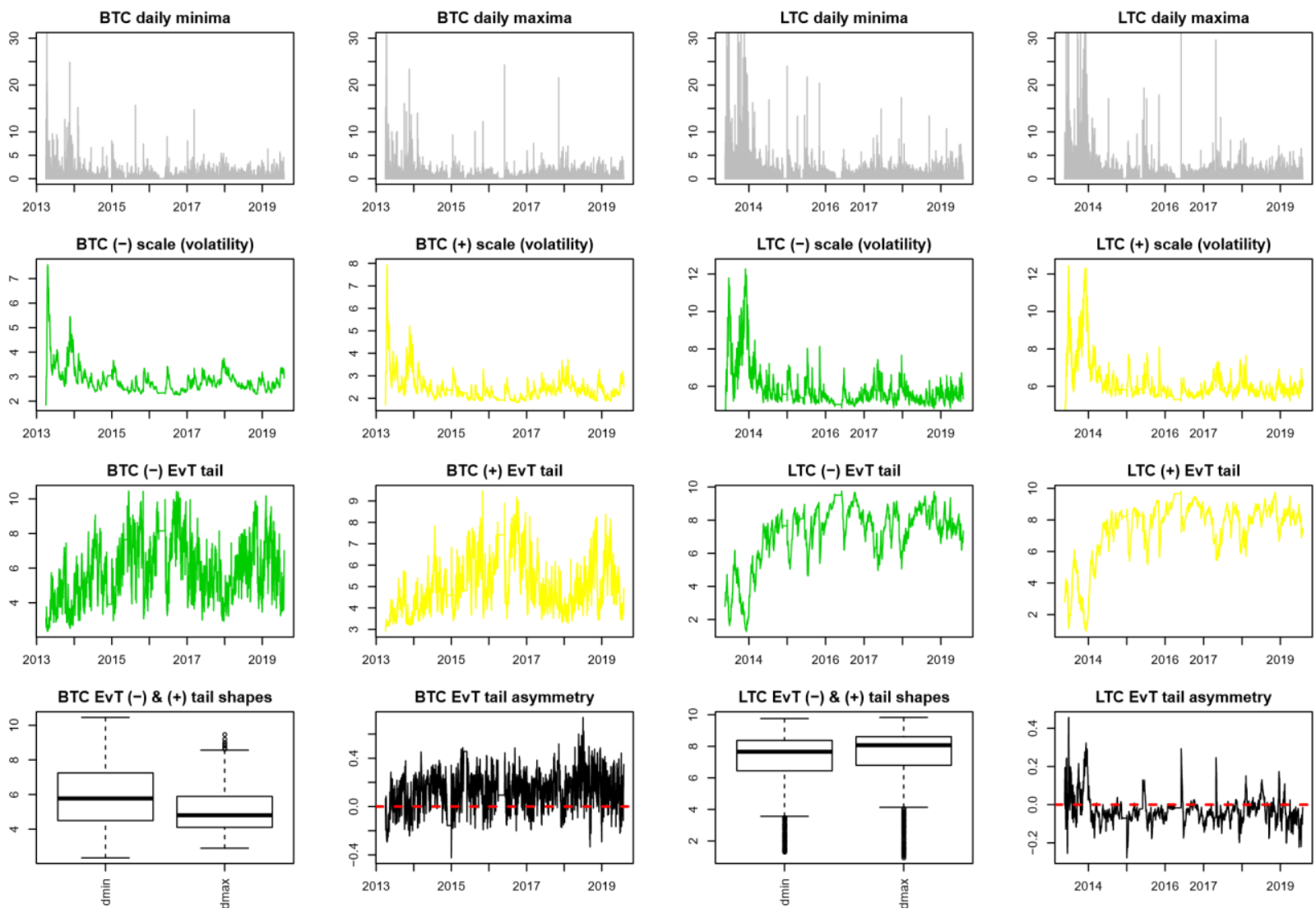
Figure 2

ETH and XRP Negative (Minima) and Positive Daily Maxima, EVT Time-varying Scale Parameter, Tail Indices, and Tail Asymmetry Measure
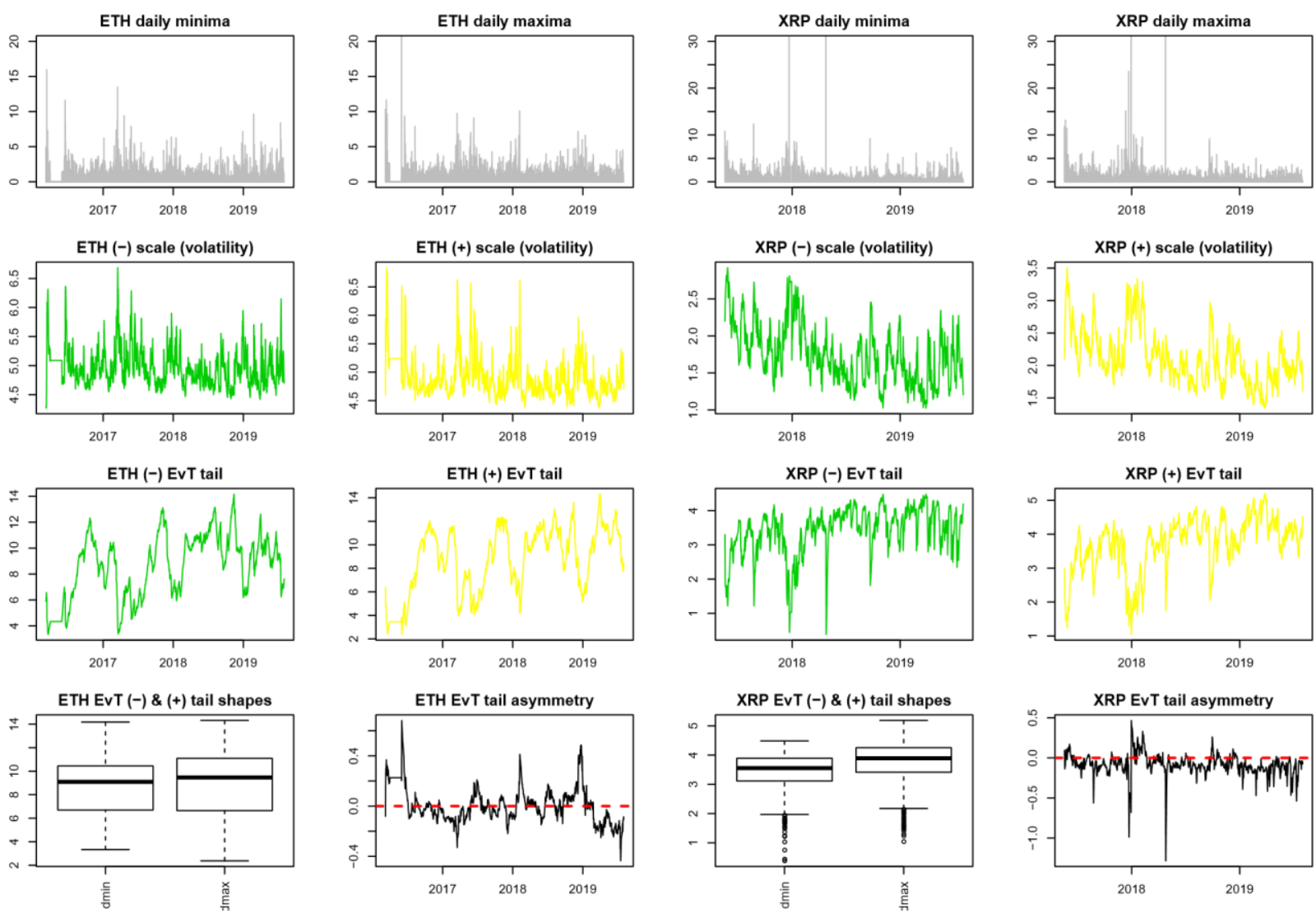
Figure 3

Bitstamp Cryptocurrency Exchange BTC Robustness Check Run
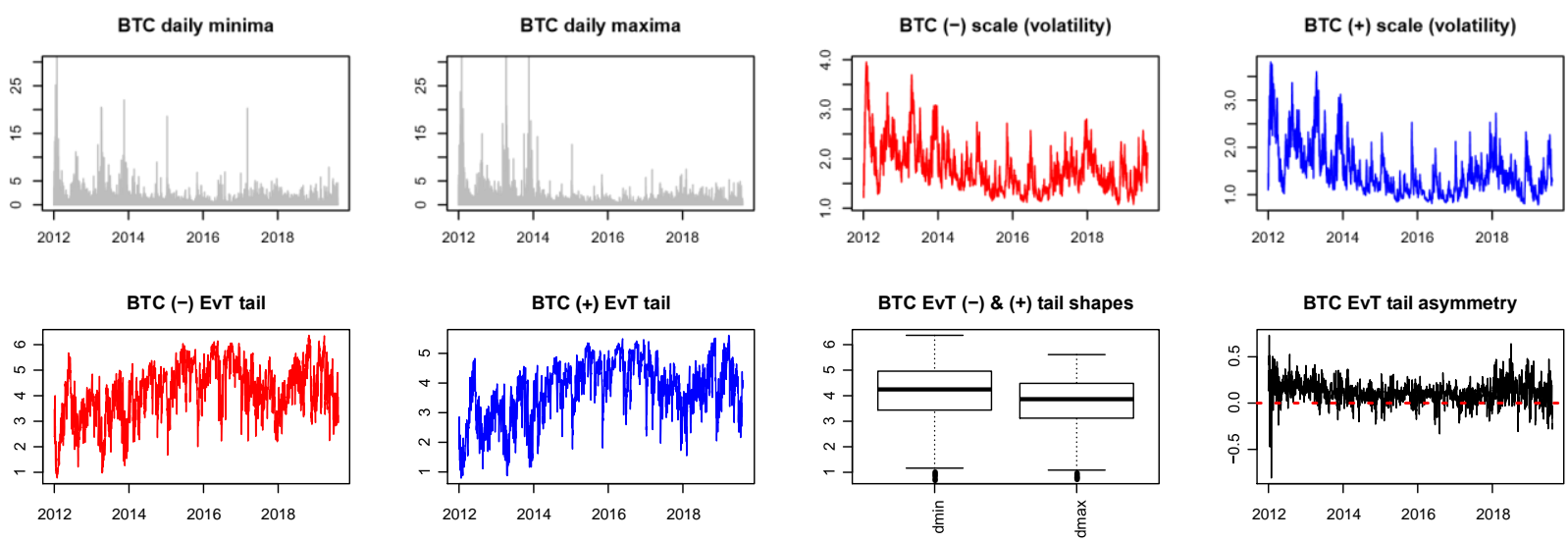